\title{
Modulation of EEG spectral edge frequency during patterned pneumatic oral stimulation in preterm infants
}

\author{
Dongli Song', Priya Jegatheesan', Sunshine Weiss', Balaji Govindaswami', Jingyan Wang², Jaehoon Lee ${ }^{3}$, Austin Oder ${ }^{2}$ \\ and Steven M. Barlow ${ }^{2}$
}

BACKGROUND: Stimulation of the nervous system plays a central role in brain development and neurodevelopmental outcomes. Thalamocortical and corticocortical development is diminished in premature infants and correlated to electroencephalography (EEG) progression. The purpose of this study was to determine the effects of orocutaneous stimulation on the modulation of spectral edge frequency $f=90 \%$ (SEF-90), which is derived from EEG recordings in preterm infants.

METHODS: A total of 22 preterm infants were randomized to experimental and control conditions. Pulsed orocutaneous stimulation was presented during gavage feedings begun at $\sim 32$ wk postmenstrual age. The SEF-90 was derived from twochannel EEG recordings.

RESULTS: Compared with the control condition, the pulsed orocutaneous stimulation produced a significant reorganization of SEF-90 in the left $(P=0.005)$ and right $(P<0.0001)$ hemispheres. Notably, the left and right hemispheres showed a reversal in the polarity of frequency shift, demonstrating hemispheric asymmetry in the frequency domain. Pulsed orocutaneous stimulation also produced a significant pattern of short-term cortical adaptation and a long-term neural adaptation manifested as a $0.5 \mathrm{~Hz}$ elevation in SEF-90 after repeated stimulation sessions.

CONCLUSION: This is the first study to demonstrate the modulating effects of a servo-controlled oral somatosensory input on the spectral features of EEG activity in preterm infants.

$\mathbf{T}$ he astonishing capabilities of the human brain arise from intricate connectivity patterns among its billions of interacting brain cells. The specific patterns of connectivity are forged by the unique interaction between a person's genes and the local environment (1). Injury or deprivation of environmental input occurring at specific stages of postnatal life can dramatically reshape brain circuit development. For premature infants, the perinatal insults associated with premature birth and extrauterine environment significantly disrupt normal thalamocortical and corticocortical development (2). Extremely premature infants endure an extensive course of respiratory support and frequent adverse stimulation during their neonatal intensive care unit (NICU) stay. The invasiveness of intubation, oxygen supplementation procedures, and prolonged nasogastric feeding tube placement disrupt preterm infant precious sensory and motor experiences during a critical period of brain development, leading to maladaptive behaviors and abnormal development. Routine caregiving procedures (e.g., manipulation, diaper changes, endotracheal tube suctioning, repositioning) in critically ill preterm infants are associated with major circulatory fluctuations in cerebral hemodynamics that are underdetected by current bedside monitoring (3). However, introducing a developmentally appropriate NICU environment and stimulation has the potential to protect normal brain development $(4,5)$. Collectively, the effects of prematurity on neural development underscore the need for bedside brain monitoring tools (e.g., electroencephalography (EEG), near-infrared spectroscopy) to assess neurologic status and better understand the effects of stimulus modalities and caregiver manipulations on brain activity (6).

Defining normality of electrocortical activity represents a significant challenge in the NICU (7). Reduced-montage twochannel EEG is becoming a common technique to monitor and map brain maturation and assess neurological status in preterm infants. Amplitude-integrated EEG (aEEG) reflects integrated brain activity and allows for time-compressed continuous electrocortical monitoring at bedside. Two pairs of surface electrodes (C3-P3 and C4-P4) are placed on the scalp over the centroparietal cortices, proximal to forelimb and orofacial sensorimotor cortical representations for aEEG recording. The aEEG shows many electrocortical features, including the type of background activity (discontinuous/continuous), interburst intervals or burst rate, cyclic variation in the background activity corresponding to sleep-wake cycling, upper and lower amplitude margins, amplitude bandwidth, spectral composition, and the presence of seizure patterns (8).

The spectral signature of brain activity, including exogenous and autogenic frequency modulation (e.g., neonate state changes, external stimulation) and rhythmic electrocortical activity (resting state network dynamics), provides

'Division of Neonatology, Department of Pediatrics, Santa Clara Valley Medical Center, San Jose, California; ${ }^{2}$ Department of Speech-Language-Hearing: Sciences and Disorders, Programs in Neuroscience and Bioengineering, University of Kansas, Lawrence, Kansas; ${ }^{3}$ Center for Research Methods and Data Analysis, University of Kansas, Lawrence, Kansas. Correspondence: Dongli Song (dongli.song@hhs.sccgov.org)

Received 24 March 2013; accepted 16 June 2013; advance online publication 20 November 2013. doi:10.1038/pr.2013.179 


\section{Articles | songet al.}

clinicians and neuroscientists with a window into the integrity and maturation of the human nervous system in the frequency domain. Spectral edge frequency $f_{c}=90 \%$ (SEF-90), defined as the frequency below which $90 \%$ of the power in a spectrum resides, is thought to reflect cerebral maturation. Application of the SEF measure in healthy newborns revealed a significant positive correlation with gestational age (GA) and varied with behavioral state and brain loci (9). Between $\sim 30$ and $40 \mathrm{wk}$ GA, whole-brain SEF values increased from 5.4 to $12 \mathrm{~Hz}$. Automated spectral power analyses showed that brain maturation is indicated by a significant shift from the lower to the higher EEG frequencies with advancing postmenstrual age (PMA) and that gyral development in the parietotemporal cortex precedes that of other areas $(10,11)$. The evolution of preterm brain maturity as seen by EEG correlates with progression of gyration $(12,13)$.

To date, most studies using processed forms of EEG to examine preterm brain cortical activity have been aimed at mapping developmental features of maturation and/or pathologic brain activity. An additional consideration in critical care of the preterm infant is the local environment in the NICU, including the role of uni- or multimodal stimulation in brain development and neurodevelopmental outcomes. The aim of this study was to determine the effects of highly controlled (pneumatic servo) pulsed orocutaneous stimulation, presented during gavage feedings begun at $\sim 32 \mathrm{wk}$ PMA, on the modulation of cerebral activity in the frequency domain among medically stable preterm infants.

\section{RESULTS}

\section{Spectral Edge Frequency}

The presence of the pulsed pneumatic orocutaneous stimulation produced a visible reorganization of aEEG signal margins as shown in the time domain for the two examples in Figure 1. During somatosensory stimulation, a shift in lower margins is apparent for each infant sampled at $32 \mathrm{wk}$ PMA. Fourier analysis revealed that the pulsed orocutaneous stimulation produced a significant reorganization of SEF-90 when compared with the blind pacifier condition in the left $(P=0.005)$ and right $(P<0.0001)$ hemispheres (Tables 1 and 2).

\section{Asymmetry}

Based on the mixed model for repeated measures, the adjusted means for SEF-90 showed a reversal in the polarity of frequency shift between the left and right hemispheres (Table 1). The adjusted means for the SEF-90 in the left hemisphere were 10.79 and $11.16 \mathrm{~Hz}$ for the patterned pneumatic orocutaneous and blind pacifier conditions, respectively. The adjusted means for the SEF-90 in the right hemisphere were 11.38 and 10.87 $\mathrm{Hz}$ for the patterned pneumatic orocutaneous and blind pacifier conditions, respectively. This demonstrates an intriguing hemispheric asymmetry in EEG frequency domain.

\section{Cortical Adaptation}

A significant pattern of cortical adaptation in the SEF measure was apparent in the present cohort for both the blind and pulsed orocutaneous pacifier stimulation conditions, and a significant difference was apparent between the two conditions as well (Figure 2). The response to stimulation on SEF in P5 and P7 was progressively attenuated compared with P3 in both the groups. The 5.5-min periods of "no-stimulation" in the experimental infants showed a significant aftereffect in the SEF-90, especially following the first- and second-stimulation epochs. The SEF-90 aftereffect was not apparent to the same degree in the control infants. SEF was elevated overall by $\sim 0.5 \mathrm{~Hz}$ in infants in the experimental group, as compared with control infants, who did not receive the pulsatile stimulation. Comparing mean values of SEF-90 in the first stimulation session (Figure 3 ) showed that the SEF-90 baseline (periods 1 and 2) was not significantly different between sham and pulsed infant groups $(P=0.898)$. Moreover, the potent effects of the pulsatile orocutaneous stimulus are evident in the divergence of sham and pulsed pacifier stimulation functions during the infant's first recording session.

\section{DISCUSSION}

Cerebral and subcortical maturation and activity-dependent changes in the newborn are complex processes and are reflected in EEG measures (14). The 24-32 wk gestational period is characterized by the prominence of the transient cortical subplate, which expands to approximately five times the size and thickness of the cortical plate $(15,16)$. The subplate plays a critical role in the development of thalamocortical and corticocortical connections and contributes directly and indirectly to EEG activity. The subplate serves as a potential site for the mechanisms underlying the spontaneous activity transients, as they are dominated by low-frequency waves with superimposed high-frequency components, a feature of early preterm EEG (17).

\section{Diminished Thalamocortical Development in Prematurity}

Subcortical inputs from the brainstem and thalamus provide an essential source of patterned activity to the developing neocortex (18). Diffusion tensor imaging has revealed significantly diminished preterm thalamocortical connectivity (19). These anatomical findings are corroborated by hemodynamic measures of connectivity in functional magnetic resonate imaging. Compared with full-term infants, very preterm infants, scanned at term equivalent age, exhibit reduced connectivity between the cerebral cortex and thalamus (20). Spectral EEG reflects complex processes related to the maturation of cerebral and subcortical pathways modulated by activity and experience. SEF has been shown to negatively correlate with the degree of white matter injury in premature infants (21).

Disruption of the thalamocortical system, correlated in EEG progression, represents a major component of preterm brain injury $(22,23)$ and negatively impacts long-term neurodevelopmental outcomes. In normal infants, resting cortical activity is characterized by a distinct spectral peak in the a range (8-13 $\mathrm{Hz}$ ). Slowing of this oscillatory peak toward the upper theta band $(6-8 \mathrm{~Hz})$ contributes to altered thalamocortical dynamics, which are associated with neurologic and neuropsychiatric 

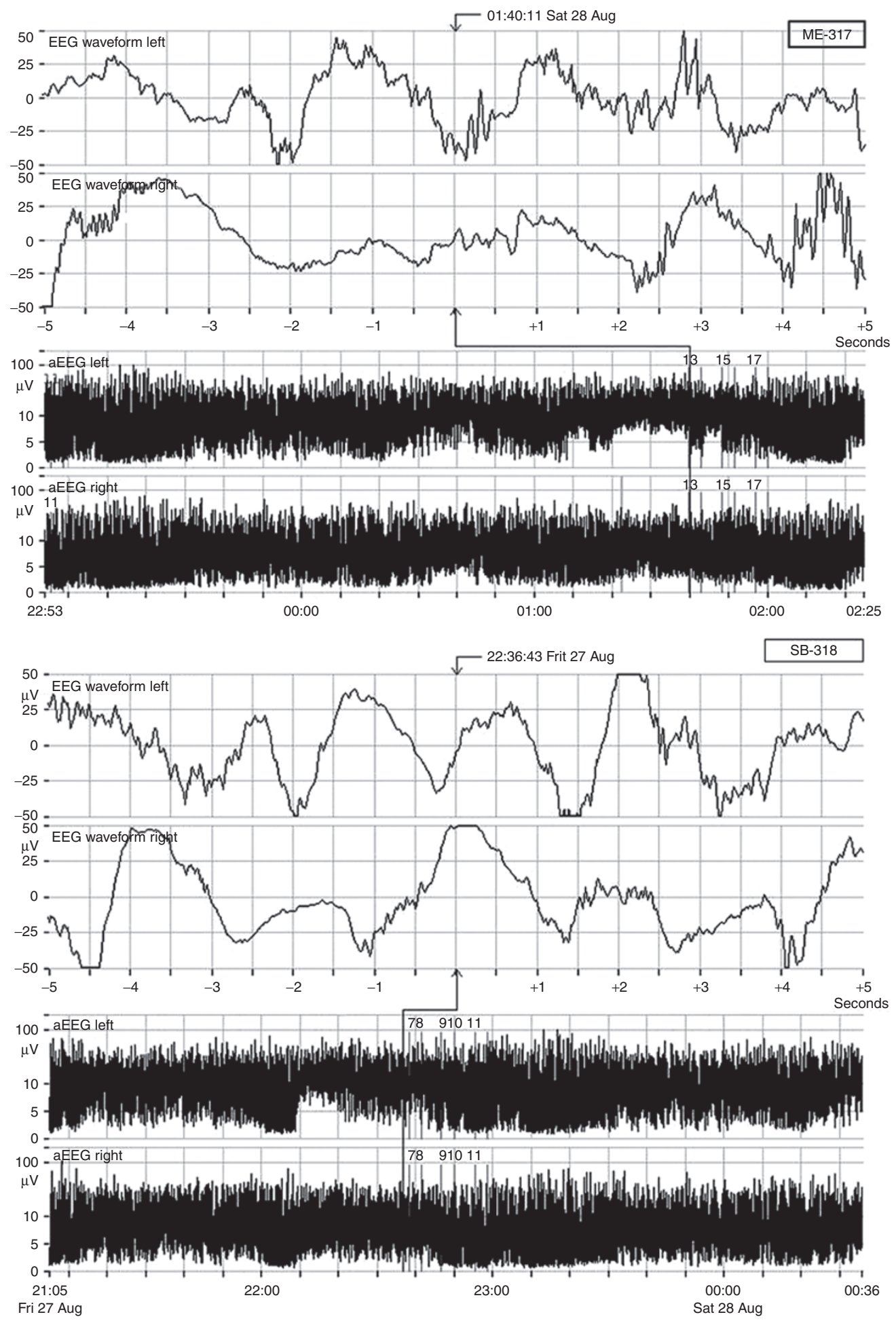

Figure 1. Examples of electroencephalography (EEG)/amplitude-integrated EEG (aEEG) using the C3-P3 and C4-P4 electrode montage on two preterm infants recorded at $32 \mathrm{wk}$ postmenstrual age. Three-min orocutaneous stimulation epochs are indexed by vertical cursors in the aEEG record (upper panel: 13, 15, and 17 and lower panel: 7, 9, and 11). Note the presence of aEEG modulation during pulsed orocutaneous stimulation. Wake-sleep cycles preceding orocutaneous stimulation periods are present.

pathology (24). Thalamic input contributes to cortical oscillatory activity that is critical for perception and cognition (25). Magnetoencephalography was used in a controlled study of global spectral activity in 11 school-age children ( $7.5 \mathrm{y}$ of age) born very preterm ( $\leq 32 \mathrm{wk}$ gestation) without major intellectual or neurological impairment (24). The very preterm children exhibited a slowing of peak frequency toward the theta band over their bilateral frontal cortex and a reduction 
Table 1. Mixed model adjusted means

\begin{tabular}{|c|c|c|c|c|c|c|c|c|c|c|c|}
\hline Group & \multicolumn{2}{|c|}{ Exp-ON } & \multirow{2}{*}{\multicolumn{2}{|c|}{$\begin{array}{c}\text { Exp-OFF } \\
\begin{array}{c}\mathrm{P} 1, \mathrm{P} 2, \mathrm{P} 4, \mathrm{P} 6, \mathrm{P} 8, \\
\text { and } \mathrm{P9}\end{array}\end{array}$}} & \multirow{2}{*}{\multicolumn{2}{|c|}{$\begin{array}{c}\text { Cont-ON } \\
\text { P3, P5, and P7 }\end{array}$}} & \multirow{2}{*}{\multicolumn{2}{|c|}{$\begin{array}{c}\text { Cont-OFF } \\
\begin{array}{c}\mathrm{P} 1, \mathrm{P} 2, \mathrm{P} 4, \mathrm{P} 6, \mathrm{P} 8 \\
\text { and } \mathrm{P} 9\end{array}\end{array}$}} & \multirow{2}{*}{\multicolumn{3}{|c|}{ Group effect }} \\
\hline Periods & \multicolumn{2}{|c|}{ P3, P5, and P7 } & & & & & & & & & \\
\hline SEF-90 Left & 10.79 & 0.28 & 10.99 & 0.28 & 11.16 & 0.29 & 11.08 & 0.28 & 5.21 & 3,30 & 0.005 \\
\hline SEF-90 Right & 11.38 & 0.2 & 11.27 & 0.19 & 10.87 & 0.2 & 10.77 & 0.19 & 21.52 & 3,30 & 0 \\
\hline
\end{tabular}

P1-P9 represent nine sequential periods.

Cont-OFF, controls blind pacifier is removed from the infant's mouth; Cont-ON, controls blind pacifier stimulation; Exp-OFF, experimental pacifier is removed from the infant's mouth; Exp-ON: experimental pacifier with pneumatic pulse stimulation; SEF-90, spectral edge frequency $f_{c}=90 \%$.

Table 2. Post hoc pairwise comparison

\begin{tabular}{|c|c|c|c|c|c|c|}
\hline \multirow[b]{2}{*}{ Variable } & \multicolumn{6}{|c|}{ Bonferroni-adjusted $P$} \\
\hline & G1-G2 & G1-G3 & G1-G4 & G2-G3 & G2-G4 & G3-G4 \\
\hline SEF-90 Left & 0.062 & 0.007 & 0.014 & 0.388 & 1.000 & 1.000 \\
\hline SEF-90 Right & 0.947 & 0.000 & 0.000 & 0.001 & 0.000 & 1.000 \\
\hline
\end{tabular}

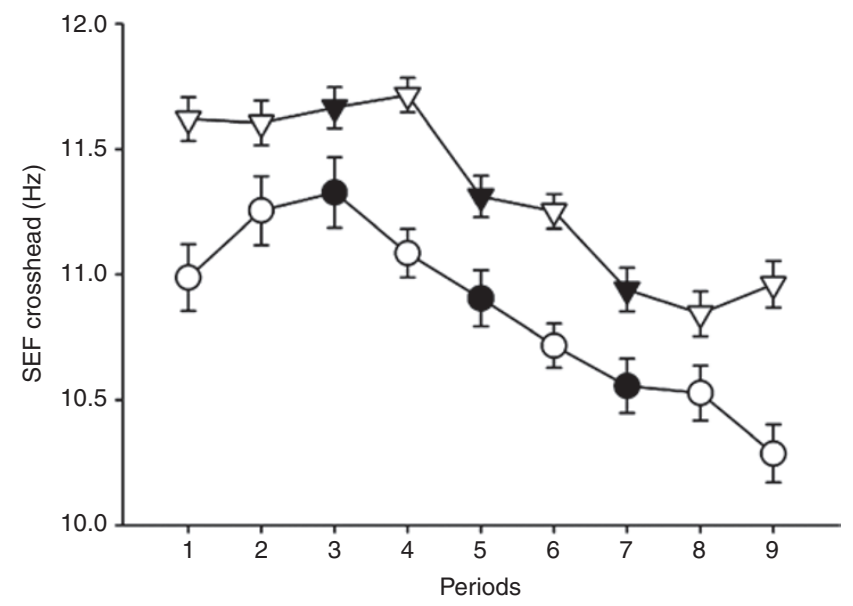

Figure 2. Interval plot of spectral edge frequency $f=90 \%$ (SEF-90) for crosshead (C3-P3 and C4-P4) montage for blind "sham" pacifier (circles) vs. the "pulsed" pacifier (triangles). Data from all stimulation sessions. Filled symbols represent stimulation conditions. Open symbols indicate periods when neither sham nor pulsed pacifier was in the baby's mouth. Contiguous electroencephalography periods (data blocks) labeled 1-9 (periods). All periods are $3 \mathrm{~min}$ in duration, except periods 4 and 6 that are $5.5 \mathrm{~min}$ in duration.

in the $\alpha$ band power over their bilateral frontal and temporal cortices, suggesting that mildly dysrhythmic thalamocortical interactions may contribute to altered spontaneous cortical activity in children born very preterm.

\section{SEF Modulation}

Modulation of EEG spectra during and after somatosensory stimulation as observed in this study reflects the dynamics of thalamocortical excitation. Pulsed pneumatic orocutaneous stimulation produces synchronous oral mechanoreceptive afferent activity in the maxillary (V2) and mandibular (V3) divisions

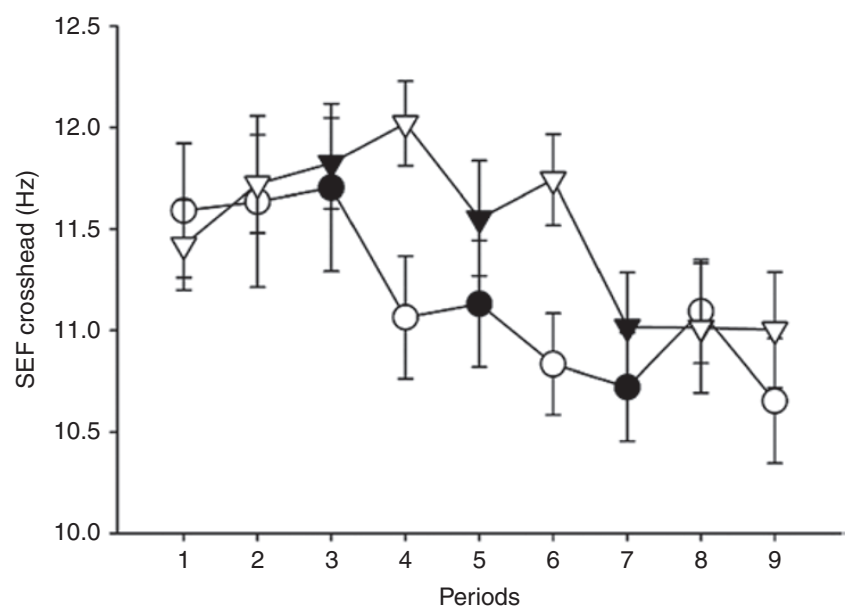

Figure 3. Plot of the spectral edge frequency $f_{\mathrm{c}}=90 \%$ (SEF-90) for crosshead (C3-P3 and C4-P4) montage for blind "sham" pacifier (circles) vs. the "pulsed" pacifier (triangles). Data from first stimulation session. Filled symbols represent stimulation conditions. Open symbols indicate periods when neither sham nor pulsed pacifier was in the baby's mouth. Contiguous electroencephalography periods (data blocks) labeled 1-9 (periods). All periods are 3 min except periods 4 and 6, which are 5.5 min in duration.

of the trigeminal nerve. Based on the observed upregulation of SEF-90 during and immediately after pulsed orocutaneous stimulation, we hypothesize that the frequency of electrocortical activity is influenced by bilateral trigeminal-thalamocortical input. The modulation of the spectra may reflect a form of plasticity, adaptation that facilitates increased rates of cortical activity for several minutes after the stimulus is removed.

In preterm infants, aEEG matures predominantly with PMA (26). We demonstrate here that pulsed orocutaneous stimulation to the lip and mouth produces changes in SEF-90. Such orocutaneous stimulus has been shown to improve sucking and feeding skills in preterm infants (5). Other early developmentally appropriate experiences, such as gentle care, dim lights, rest periods, flexion positioning, and holding, improve the development of brain structure and function, as evidenced by EEG spectral coherence and neurobehavioral function (4). Massage of preterm infants increases aEEG amplitudes and delta, alpha, theta, and beta EEG frequency domains (27). Skin-to-skin contact facilitates brain maturation in preterm infants, resulting in a more mature sleep pattern, improved respiratory regularity, and less spectral beta activity (28). 


\section{SEF Asymmetry}

We demonstrate significant asymmetry in $32 \mathrm{wk}$ PMA infant cortical SEF during oral somatosensory stimulation. There is an abundance of evidence to support anatomic and functional cortical asymmetry in early life (29). However, obtaining a complete understanding of structural and functional hemispheric asymmetries in early life remains one of the main challenges in developmental neuroscience. One example of functional asymmetry is the right-sided lateralization of pitch processing detected at $35 \mathrm{wk}$ PMA in infants born at greater than $30 \mathrm{wk}$ gestation (29). Macroscopic left-right differences are present from the fetal life and onward (30). The developmental time scale also differs between the cerebral hemispheres. The superior frontal gyrus, the superior temporal gyrus, and Heschl's gyrus appear 1-2 wk sooner in the right hemisphere than those in the left hemisphere (31), whereas other evidence has shown that the planum temporale and Heschl's gyrus are larger in the left hemisphere in fetuses and infants (31). Dubois et al. (13) reported early fetal third-trimester brain rightward morphological asymmetry, with the right temporal sulcus being larger than the left. Neonates, in contrast to adults, tend to have larger gray and white matter volumes in the left hemisphere (32). In infants aged 3-11 mo, myelination asymmetries were observed (33), with slower myelination in the right cerebellum and left cerebrum relative to the contralateral structures. Dubois et al. (30) used diffusion tensor imaging and spatial localization methods to demonstrate early leftward asymmetries in the arcuate fasciculus and corticospinal tract, suggesting that early macroscopic geometry, microscopic organization, and white matter bundle maturation may be related to later functional lateralization.

Among the most intriguing lateralized functions in humans are hand preference and speech-language function. Studies during late gestation and shortly after birth are needed to understand how these asymmetries arise during brain growth and development and to determine if left-right differences in brain structure are the source or the consequence of functional lateralization (30).

\section{Cortical Adaptation}

This study showed cortical adaptation in the SEF measure. Sensory adaptation, defined as response attenuation to repeated stimulation, can be short term or long term and can occur in any anatomic location along the neurosensory pathway, from peripheral mechanosensors to the cerebral cortex. Cortical adaptation to sustained sensory input, as used in the present study, is regarded as one of the most ubiquitous forms of shortterm plasticity. This mechanism improves spatial and temporal resolution of sensory events, which in turn improves motor learning and development of categorical perception for higher cognitive and communicative function. Neural adaptation can be observed in either the amplitude or frequency domain. In terms of frequency-following capabilities, the rate of neural response adaptation tends to increase as one proceeds from the periphery to the S1/S2 cortex. A recent functional neuroimaging study using the same pulsed pneumatic orocutaneous stimulus demonstrated adaptation in neuromagnetic response magnitude in S1 and S2 in young adults (34).

We observed two interesting phenomena in the cerebral SEF-90 measures that merit further discussion: an overall upward shift in the SEF-90 (Figures 2 and 3) and a persistent SEF aftereffect once the pulsed orocutaneous stimulus was removed. These effects may reflect different underlying mechanisms of cortical adaptation in the preterm brain. We reasoned that the overall upshift in SEF-90 in the experimental group is likely due to long-term neural adaptation, indicative of the collective effects of repeated stimulation over several sessions and days (Figure 2). To test this notion in the present data set, an additional analysis of the SEF modulation among sham and experimental group infants was completed to rule out the possibility that their baseline SEF profiles were somehow different between groups at the beginning of the study. As shown in Figure 3, the baseline SEF profiles, indicated by periods 1 and 2 for sham and experimental group infants, were not significantly different, indicating that the observed overall difference in SEF-90 between the two groups developed after experience of the different stimulus conditions (sham vs. pulsed orocutaneous).

A significant aftereffect or persistence in SEF-90 adaptation also was observed in the preterm infants who received the pulsed orocutaneous stimulation. This represents a relatively short-term form of cortical adaptation to our servo-controlled orocutaneous experience and may serve as a biomarker of brain maturation and may allow us to map the effects of sensory intervention therapies. In the mature brain, sustained sensory stimulation leads to transformations in the thalamocortical encoding that impact the nature of information conveyed about the sensory stimuli. Changes in midlayer cortical neuron spiking activity reflect a switch in their role with adaptation, from coincidence detectors (tuned for stimulus detection in a nonadapted state) to integrators (tuned for stimulus discrimination after adaptation) (35). With repetitive stimulation, a dynamic cortical inhibitory mechanism shapes the initial activity into a stimulus-specific spatial pattern of columnar assemblies (36). Lateral interactions between adjacent columns are the result of intrinsic inhibitory and excitatory effects, which lead to a differential modification (across columns) of the membrane potential that can last for as long as several seconds.

\section{Conclusion}

The present study describes the application of a new oral somatosensory stimulation regimen with bedside EEG monitoring, an emerging NICU technology for detecting the spectral signature of electrocortical activity. Features of this approach are highly effective for monitoring preterm infants, including (i) the precocial nature of trigeminal orofacial cutaneous sensitivity $(37,38)$ and (ii) high cortical magnification factor for orofacial sensory representation in S1/S2 (39). Thus, the dual-channel aEEG/EEG montage, situated over the infant's lateral cerebral convexity, allows quantitative mapping of the effects of a highly controlled somatosensory input on 
a
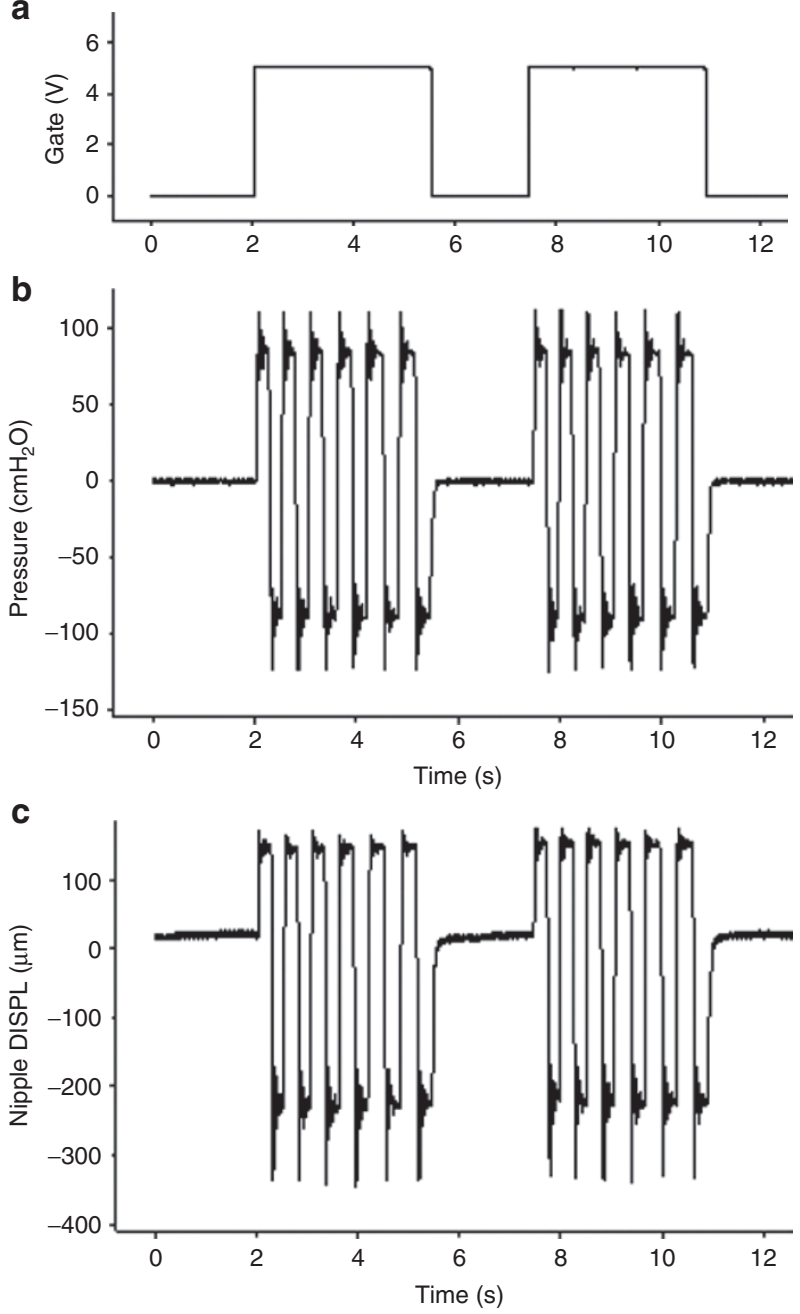

Figure 4. Frequency-modulated orocutaneous stimulus burst pattern consisting of six pulses followed by a 2-s pause period. A servo-controlled microprocessor (a) generates the signal gating function to (b) dynamically "charge" the intraluminal pressure of the silicone pacifier, (c) resulting in rapid changes in pacifier geometry.

SEF-90 electrocortical activity. This work lays a foundation for further elucidating mechanisms of short- and long-term neural adaptation and maturation of thalamocortical networks in preterm infants.

\section{METHODS}

\section{Participants}

This study was approved by Santa Clara Valley Medical Center Human Subjects Internal Review Board, and parental consent was obtained for all subjects before enrollment. A total of 22 healthy preterm infants, (16 males and 6 females) with a mean GA of $28.6 \mathrm{wk}$ ( $\mathrm{SD}=2.1)$, birth weight of $1,230 \mathrm{~g}(\mathrm{SD}=338)$, and PMA at test of $32.2 \mathrm{wk}(\mathrm{SD}=1.09)$, were enrolled in the study.

\section{Inclusion Criteria}

Preterm infants with GA of $24-32 \mathrm{wk}$ and at least $28 \mathrm{wk}$ PMA at the time of enrollment were included in the study.

\section{Exclusion Criteria}

Infants with chromosomal abnormalities, multiple congenital anomalies, or any major congenital anomalies were excluded from the study. Infants with a history of severe intraventricular hemorrhage,
Table 3. Stimulation schedule

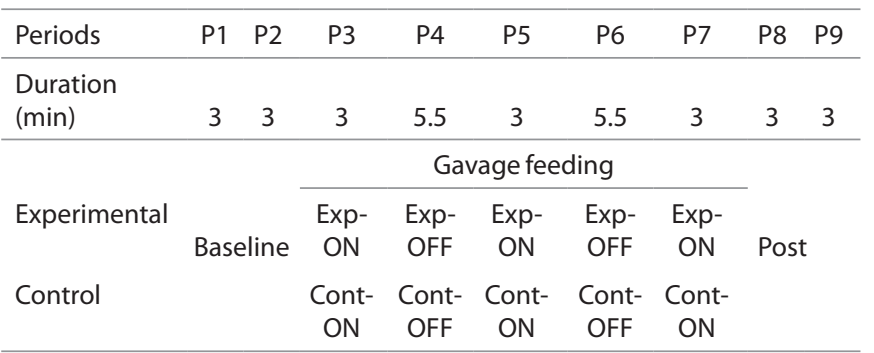

Nine sequential periods are indicated by P1-P9. The pulsed or blind "sham" pacifier is presented to the infant during P3, P5, and P7. The pacifier is not in the infant's mouth during $\mathrm{P} 1, \mathrm{P} 2, \mathrm{P} 4, \mathrm{P} 6, \mathrm{P} 8$, and $\mathrm{P} 9$.

Cont-ON, controls blind pacifier stimulation is presented to the infant; Cont-OFF, pacifier is removed from the infant's mouth; Exp-OFF, pacifier is removed from the infant's mouth; Exp-ON, experimental pacifier with pneumatic pulse stimulation is presented to the infant.

necrotizing enterocolitis ( $\geq$ stage III), vocal cord paralysis, seizures, or meningitis, and infants who were nippling all feeds at the time of enrollment were also excluded.

\section{Experimental Design}

Study infants were randomly assigned to two groups, including those who received pulsed orocutaneous stimulation (experimental) and those who did not (control). The pulsed orocutaneous stimulation was delivered by a servo-controlled pneumatic amplifier (Ntrainer System, Innara Health, Shawnee, KS) specifically designed to transmit repeating pneumatic pulse trains to the soft tissues of the infant's lips-anterior tongue-intraoral mucosa-jaw through a regular (green) Soothie silicone pacifier (Philips AVENT Pacifier; Philips Healthcare, Andover, MA) (5). This six-cycle orocutaneous stimulus burst was frequency modulated (interpulse cycle periods of 510,526,551,580, and $626 \mathrm{~ms}$ ) with an intertrain interval of 2 -s and $50-\mathrm{ms}$ pulse width (Figure 4). The pressure rise-fall time (10-90\% intercepts) was $31 \mathrm{~ms}$, and the resultant displacement at the pacifier-lip/tongue tissue interface was $\sim 400 \mu \mathrm{m}$. Three-min pneumatic orocutaneous stimulation periods were interleaved with 5.5min pause periods, where the pacifier was removed from the infant's mouth (Table 3). The control infants received a sham stimulation program in which infants were offered the same type of Soothie pacifier without patterned stimulation (blind pacifier). The staging of a single stimulation session was given concurrently with gavage feeds. Infants had up to three daily sessions at routine feedings scheduled every $3 \mathrm{~h}$. Infants were swaddled in a blanket with hands positioned at midline, in a quiet-awake to drowsy state during stimulation.

\section{EEG Recording and Signal Processing}

Four neonatal hydrogel sensors (Natus Medical, San Carlos, CA) were placed in the $\mathrm{C} 3, \mathrm{C} 4, \mathrm{P} 3$, and $\mathrm{P} 4$ positions according to the international 10-20 system for EEG monitoring. EEG signals were recorded on a BRM3 monitor (Brainz; Natus Medical) for up to $4 \mathrm{~d}$ beginning at $\sim 32$ wk PMA (Figure 5). The right- and left-side EEG signals were amplified (Gain $=5 \mathrm{~K})$, bandpass-filtered, using a first-order high-pass filter $\left(f_{\mathrm{c}}=-3 \mathrm{~dB}\right.$ at $\left.1 \mathrm{~Hz}\right)$ and a fourth-order low-pass Butterworth $(f=-3 \mathrm{~dB}$ at $50 \mathrm{~Hz})$ filter, and digitized at a sampling rate of $256 \mathrm{~Hz}$. Brainz Analyze Research (v1.5) software (Natus Medical, San Carlos, CA) was used to derive the SEF-90 at 1-min intervals. These electroencephalographic measures were derived from nine sequential epochs (data blocks), spanning $32 \mathrm{~min}$ each, and centered over the pulsed orocutaneous or the blind pacifier conditions. A total of 1,620 EEG blocks were analyzed among the 22 infants. The average number of orosensory sessions sampled per infant was $8.18(\mathrm{SE}=1.09)$. Portions of recordings were excluded from analysis if electrode impedance exceeded $10 \mathrm{k} \Omega$, or if there was gross movement, electrical noise artifact, or asymmetry of baseline between the two channels (14).

\section{Statistical Analyses}

Mixed models for repeated measures were used to compare the EEG SEF-90 measures among four stimulus conditions, including 


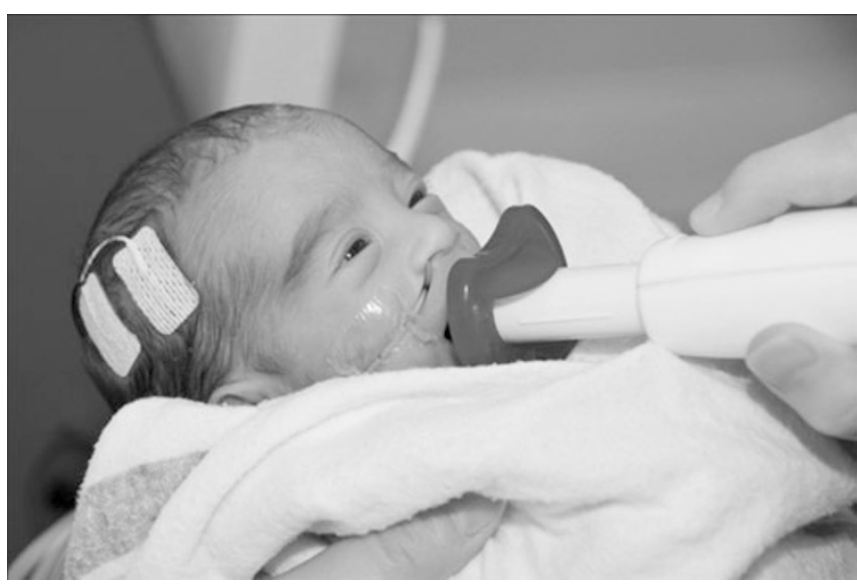

Figure 5. Preterm infant with amplitude-integrated electroencephalography and pneumatically pulsed stimulation through a silicone pacifier coupled to the handpiece of the Ntrainer System (Innara Health, Shawnee, KS).

(i) pacifier with pneumatic pulse stimulation (Exp-ON), (ii) pacifier without pneumatic pulse stimulation (Exp-OFF), (iii) controls with blind (nonpulsitile) pacifier stimulation (Cont-ON), and (iv) controls without blind (nonpulsitile) pacifier stimulation (Cont-OFF). Adjusting for the infants' GA and birth weight, mixed models estimated the stimulus effect on each outcome via the use of restricted maximum likelihood estimator and compound symmetric error covariance structure. When the stimulus effect was significant at $a=0.05$, adjusted means were pairwise compared using a Bonferronicorrected $P$ value. All analyses were conducted using SAS 9.3 (SAS Institute, Cary, NC).

\section{ACKNOWLEDGMENTS}

We express our deepest gratitude to the extremely dedicated NICU staff and families of our preterm participants at Santa Clara Valley Medical Center.

\section{STATEMENT OF FINANCIAL SUPPORT}

This study was supported in part by grants from Santa Clara County First Five (San Jose, CA), Valley Medical Center Foundation (San Jose, CA), National Institutes of Health (NIH) (Bethesda, MD; grant R01 DC003311 to S.M.B. and grant P30 HD02528), and the Sutherland Family Foundation (Lawrence, KS).

Disclosure: None of the authors has a direct financial relation with Philips, Natus, Brainz Analyze Research, or SAS statistical software. Barlow is the inventor of the Ntrainer System, which is licensed by the University of Kansas to Innara Health (Shawnee, KS).

\section{REFERENCES}

1. Penn AA, Shatz CJ. Brain waves and brain wiring: the role of endogenous and sensory-driven neural activity in development. Pediatr Res 1999;45(4 Pt 1):447-58.

2. Ball G, Boardman JP, Rueckert D, et al. The effect of preterm birth on thalamic and cortical development. Cereb Cortex 2012;22:1016-24.

3. Limperopoulos C, Gauvreau KK, O'Leary H, et al. Cerebral hemodynamic changes during intensive care of preterm infants. Pediatrics 2008;122:e1006-13.

4. Als H, Duffy FH, McAnulty G, et al. NIDCAP improves brain function and structure in preterm infants with severe intrauterine growth restriction. J Perinatol 2012;32:797-803.

5. Barlow SM, Finan DS, Lee J, Chu S. Synthetic orocutaneous stimulation entrains preterm infants with feeding difficulties to suck. J Perinatol 2008;28:541-8.

6. Greisen G, Leung T, Wolf M. Has the time come to use near-infrared spectroscopy as a routine clinical tool in preterm infants undergoing intensive care? Philos Trans A Math Phys Eng Sci 2011;369:4440-51.
7. Griesmaier E, Enot DP, Bachmann M, et al. Systematic characterization of amplitude-integrated EEG signals for monitoring the preterm brain. Pediatr Res 2013;73:226-35.

8. Lena Hellstrom-Westas LSdV, Rosen I. Atlas of Amplitude-Integrated EEGs in the Newborn, 2nd edn. London, United Kingdom: Informa Healthcare, 2008.

9. Bell AH, McClure BG, McCullagh PJ, McClelland RJ. Variation in power spectral analysis of the EEG with gestational age. J Clin Neurophysiol 1991;8:312-9.

10. Niemarkt HJ, Jennekens W, Pasman JW, et al. Maturational changes in automated EEG spectral power analysis in preterm infants. Pediatr Res 2011;70:529-34.

11. Niemarkt HJ, Jennekens W, Maartens IA, et al. Multi-channel amplitudeintegrated EEG characteristics in preterm infants with a normal neurodevelopment at two years of corrected age. Early Hum Dev 2012;88:209-16.

12. Biagioni E, Frisone MF, Laroche S, et al. Maturation of cerebral electrical activity and development of cortical folding in young very preterm infants. Clin Neurophysiol 2007;118:53-9.

13. Dubois J, Benders M, Cachia A, et al. Mapping the early cortical folding process in the preterm newborn brain. Cereb Cortex 2008;18:1444-54.

14. Mizrahi EM, Hrachovy R, Kellaway P, Stockard-Pope JE. Atlas of Neonatal Electroencephalography, 3rd edn. Philadelphia, PA: Lippincott Williams \& Wilkins, 2004.

15. Kostovic I, Jovanov-Milosevic N. The development of cerebral connections during the first 20-45 weeks' gestation. Semin Fetal Neonatal Med 2006;11:415-22.

16. Kostovic I, Rakic P. Developmental history of the transient subplate zone in the visual and somatosensory cortex of the macaque monkey and human brain. J Comp Neurol 1990;297:441-70.

17. Vanhatalo S, Kaila K. Development of neonatal EEG activity: from phenomenology to physiology. Semin Fetal Neonatal Med 2006;11:471-8.

18. Grant E, Hoerder-Suabedissen A, Molnár Z. Development of the corticothalamic projections. Front Neurosci 2012;6:53.

19. Ball G, Boardman JP, Aljabar P, et al. The influence of preterm birth on the developing thalamocortical connectome. Cortex 2013;49:1711-21.

20. Smyser CD, Inder TE, Shimony JS, et al. Longitudinal analysis of neural network development in preterm infants. Cereb Cortex 2010;20:2852-62.

21. Inder TE, Buckland L, Williams CE, et al. Lowered electroencephalographic spectral edge frequency predicts the presence of cerebral white matter injury in premature infants. Pediatrics 2003;111:27-33.

22. Kostovic I, Judas M. The development of the subplate and thalamocortical connections in the human foetal brain. Acta Paediatr 2010;99:1119-27.

23. Miller SP, Ferriero DM. From selective vulnerability to connectivity: insights from newborn brain imaging. Trends Neurosci 2009;32:496-505.

24. Doesburg SM, Ribary U, Herdman AT, et al. Magnetoencephalography reveals slowing of resting peak oscillatory frequency in children born very preterm. Pediatr Res 2011;70:171-5.

25. Ribary U. Dynamics of thalamo-cortical network oscillations and human perception. Prog Brain Res 2005;150:127-42.

26. Sisman J, Campbell DE, Brion LP. Amplitude-integrated EEG in preterm infants: maturation of background pattern and amplitude voltage with postmenstrual age and gestational age. J Perinatol 2005;25:391-6.

27. Rudnicki J, Boberski M, Butrymowicz E, et al. Recording of amplitudeintegrated electroencephalography, oxygen saturation, pulse rate, and cerebral blood flow during massage of premature infants. Am J Perinatol 2012;29:561-6.

28. Scher MS, Ludington-Hoe S, Kaffashi F, Johnson MW, Holditch-Davis D, Loparo KA. Neurophysiologic assessment of brain maturation after an 8 -week trial of skin-to-skin contact on preterm infants. Clin Neurophysiol 2009;120:1812-8.

29. Mento G, Suppiej A, Altoè G, Bisiacchi PS. Functional hemispheric asymmetries in humans: electrophysiological evidence from preterm infants. Eur J Neurosci 2010;31:565-74.

30. Dubois J, Hertz-Pannier L, Cachia A, Mangin JF, Le Bihan D, DehaeneLambertz G. Structural asymmetries in the infant language and sensorimotor networks. Cereb Cortex 2009;19:414-23. 


\section{Articles | Song et al.}

31. Chi JG, Dooling EC, Gilles FH. Left-right asymmetries of the temporal speech areas of the human fetus. Arch Neurol 1977;34:346-8.

32. Gilmore JH, Lin W, Prastawa MW, et al. Regional gray matter growth, sexual dimorphism, and cerebral asymmetry in the neonatal brain. J Neurosci 2007;27:1255-60.

33. Deoni SC, Mercure E, Blasi A, et al. Mapping infant brain myelination with magnetic resonance imaging. J Neurosci 2011;31:784-91.

34. Popescu M, Barlow S, Popescu EA, et al. Cutaneous stimulation of the digits and lips evokes responses with different adaptation patterns in primary somatosensory cortex. Neuroimage 2010;52:1477-86.

35. Wang Q, Webber RM, Stanley GB. Thalamic synchrony and the adaptive gating of information flow to cortex. Nat Neurosci 2010;13 $1534-41$.
36. Whitsel BL, Favorov O, Kelly DG, Tommerdahl MA. Mechanisms of dynamic peri- and intracolumnar interactions in somatosensory cortex: stimulus specific contrast enhancement by NMDA receptor activation. In: Information Processing in Somatosensory System. Ove Franzen (ed.) London, England: Macmillan Press, 1991:353-69.

37. Humphrey T. The development of mouth opening and related reflexes involving the oral area of human fetuses. Ala J Med Sci 1968;5:126-57.

38. Barlow SM, Finan DS, Bradford PT, Andreatta RD. Transitional properties of the mechanically evoked perioral reflex from infancy through adulthood. Brain Res 1993;623:181-8.

39. Sathian K, Zangaladze A. Tactile spatial acuity at the human fingertip and lip: bilateral symmetry and interdigit variability. Neurology 1996;46: $1464-6$. 